\title{
The Characteristics of Antioxidant Activity after Liver Transplantation in Biliary Atresia Patients
}

\author{
Chih-Jen Chen, ${ }^{1}$ Kuo-Shu Tang, ${ }^{1}$ Ying-Hsien Huang, ${ }^{1}$ Chao-Long Chen, ${ }^{2}$ \\ Li-Tung Huang, ${ }^{1}$ Jiin-Haur Chuang, ${ }^{2}$ and Mao-Meng Tiao ${ }^{1}$ \\ ${ }^{1}$ Department of Pediatrics, Kaohsiung Chang Gung Memorial Hospital and Chang Gung University College of Medicine, \\ Kaohsiung 83301, Taiwan \\ ${ }^{2}$ Department of Surgery, Kaohsiung Chang Gung Memorial Hospital and Chang Gung University College of Medicine, \\ Kaohsiung 83301, Taiwan
}

Correspondence should be addressed to Mao-Meng Tiao; pc006581@yahoo.com.tw

Received 8 January 2015; Revised 29 April 2015; Accepted 30 April 2015

Academic Editor: Maria-Angeles Aller

Copyright (C) 2015 Chih-Jen Chen et al. This is an open access article distributed under the Creative Commons Attribution License, which permits unrestricted use, distribution, and reproduction in any medium, provided the original work is properly cited.

\begin{abstract}
Purpose. Cholestatic liver injury is associated with a high production of free radicals. The pathogenesis of liver injury in biliary atresia (BA) patients is largely undefined. The goal of the present study was to clarify the oxidative damage and the changes in antioxidant enzyme activities that occur during the development of BA and after liver transplantation (LT). Methods. We enrolled BA patients and control subjects and collected their clinical information. The activities of antioxidant enzymes in BA patients before LT (BA group) and after LT (LT group) were analyzed. Results. The number of mitochondrial DNA copies had increased in the LT group compared with the BA group. Similarly, the activity of glutathione peroxidase had increased in the LT group compared with the BA group. The level of glutathione was higher in the LT group than in the BA group. Malondialdehyde levels were decreased in the LT group compared with the BA group. Conclusions. These data indicate that LT is associated with increased antioxidant enzyme activities and decreased malondialdehyde levels in BA patients. The manipulation of mitochondria-associated antioxidative activity might be an important future management strategy for BA.
\end{abstract}

\section{Introduction}

Biliary atresia $(\mathrm{BA})$ is an important clinical problem involving impairments in bile flow that manifest as neonatal jaundice and can lead to progressive fibrosis and end-stage liver cirrhosis $[1,2]$. Oxidative stress and mitochondrial dysfunction are involved in the pathogenesis of chronic liver cholestasis [3]. Oxidative stress in cells or tissues is an abnormal phenomenon that occurs when the production of oxygen radicals exceeds that of the antioxidant capacity [4]. These excess free radicals damage essential cellular macromolecules, leading to abnormal gene expression and cell death [4]. Antioxidant enzymes catalyze the decomposition of these free radicals. The major antioxidant enzymes include glutathione peroxidase (GPx) and catalase (CAT), which differ in structure, tissue distribution, and cofactor requirements [5].

Disturbances in the antioxidant system could play a role in the pathogenesis of chronic liver disease [6-8] and could result in different types of liver diseases in infancy [9]. It is reported that serum oxidative stress indexes were significantly higher in the inherited metabolic diseases group than in the biliary atresia group [10]. GPx is the most important scavenger of hydrogen peroxide $\left(\mathrm{H}_{2} \mathrm{O}_{2}\right)$ in mammalian cells [11]. CAT, which is located in peroxisomes of all aerobic cells [12], is an essential enzyme in the decomposition of intracellular $\mathrm{H}_{2} \mathrm{O}_{2}$, promoting the breakdown of $\mathrm{H}_{2} \mathrm{O}_{2}$ into water and oxygen without the production of free radicals.

The copy numbers of mitochondrial DNA (mtDNA) in cells may change during cell growth and differentiation [13]. mtDNA is highly susceptible to oxidative stress that leads to mitochondrial dysfunction $[3,14]$. Previous studies suggest that an increase in the mtDNA copy number in cells is the result of a feedback mechanism that compensates for defective mitochondria that affect subsequent cell growth and morphology [14, 15]. Extrahepatic cholestatic patients 
present a significant decrease in the number of mtDNA copies compared with controls [3].

Our previous report demonstrated that the level of oxidative stress is lower after the Kasai operation. In addition, the mtDNA copy number is found to be higher after the Kasai operation [2]. To our knowledge, no study has evaluated the benefit of liver transplantation (LT) in BA patients in terms of oxidants/antioxidants. Our hypothesis is that if antioxidant levels are increased after LT $[2,10,16,17]$, then the administration of antioxidants is needed in those cases who hesitate to receive LT or in cases where a liver graft is not available from donors. Therefore, our aim was to study the oxidative status in children with BA by examining the malondialdehyde (MDA) and antioxidant enzyme levels as well as mitochondrial copy numbers.

\section{Materials and Methods}

2.1. Subjects. Participants were enrolled from the clinic at the Chang Gung Memorial Hospital, Department of Pediatrics, and liver team at the Kaohsiung Medical Center. In all patients, BA was diagnosed clinically with DISIDA scan, pathologic examination, computerized tomography, or magnetic resonance imaging findings before LT. The indications for LT among BA patients included end-stage liver disease, recurrent cholangitis, recurrent gastrointestinal bleeding, and portal hypertension. Patients who received only cyclosporine after LT were included. Those who received other immunosuppressants were excluded. Normal healthy individuals who tested negative in the allergy screen test were included as a control group. Another control group consisted of patients with idiopathic acute hepatitis. The liver tissues were randomly obtained during Kasai operation. Tissue samples from hepatitis cases were randomly obtained using a modified Minghini needle (sure cut), and biliary atresia cases who received LT had tissue samples randomly obtained at 6 months after LT. Liver tissues of normal controls were obtained from the LT donors. They were stored at $-80^{\circ} \mathrm{C}$ till assay. The total bilirubin levels and alanine transaminase (ALT) and aspartate transaminase (AST) activities were determined by a standard autoanalyzer (model 7450; Hitachi, Tokyo, Japan). The study was approved by the Ethics and Clinical Research Committee of the Chang Gung Memorial Hospital.

2.2. GPx Activity and Glutathione. GPx activity was measured using a commercially available kit (Ransel; Randox Lab, Crumlin, UK) for erythrocytes and homogenized liver tissue. Liver biopsy samples were washed in phosphate buffer at $\mathrm{pH}$ 7.4. Then, the tissue was homogenized in $5 \mathrm{~mL} / \mathrm{g}$ cold buffer, which consisted of $50 \mathrm{mM}$ Tris- $\mathrm{HCl}, \mathrm{pH} 7.5$, $5 \mathrm{mM}$ EDTA, and $1 \mathrm{nM}$ dithiothreitol. The homogenate was centrifuged at $10,000 \mathrm{~g}$ for 15 minutes at $4^{\circ} \mathrm{C}$. The supernatant was removed for assay of GPx. In the presence of glutathione reductase (GR) and NADPH, oxidized glutathione (GSSG) was immediately converted to the reduced form (GSH) with a concomitant oxidation of NADPH to NADP ${ }^{+}$. The decrease in absorbance at $340 \mathrm{~nm}$ after 1 and $2 \mathrm{~min}$ was measured.
The result obtained was expressed in units per liter (U/L) of hemolysate and was multiplied by the appropriate dilution factor to obtain the result in U/L.

2.3. Determination of CAT Activity. The plasma and liver supernatant assays were conducted on a Bradford assay (BioRad, Richmond, CA, USA) automated chemistry analyzer. The CAT activity assay kit (Cayman Chemical, Ann Arbor, MI, USA; catalog number 707002) and samples $(20 \mu \mathrm{L})$ were placed on the instrument and assayed according to the manufacturer's protocol. Liver biopsy was washed with phosphate buffer, $\mathrm{pH}$ 7.4, to remove red blood cells. The tissue was then blotted dry, weighted, and followed by homogenization in $1.5 \mathrm{~mL}$ cold buffer $(50 \mathrm{mM}$ potassium phosphate and $1 \mathrm{mM}$ EDTA, pH 7) and centrifugation at 10,000 g for 15 minutes at $4^{\circ} \mathrm{C}$. The supernatant was used for the assay. The CAT activity level was quantified spectrophotometrically at $540 \mathrm{~nm}$. The results were expressed as nanomoles per minute per milliliter of protein. Standards and blanks were assayed in duplicate and treated as samples (i.e., not placed in the calibration positions). The average of 2 measurements was used in subsequent statistical analysis of the data.

2.4. Determination of Plasma MDA Content. MDA is an end product of peroxidative decomposition of polyenoic fatty acids in the lipid peroxidation process, and its accumulation in tissues is indicative of the extent of lipid peroxidation. Plasma MDA was measured using the thiobarbituric acid reactive substances (TBARS) assay. The TBARS reagent $(1 \mathrm{~mL})$ was added to an aliquot containing $0.5 \mathrm{~mL}$ plasma, and the mixture was heated for $20 \mathrm{~min}$ at $100^{\circ} \mathrm{C}$. The antioxidant, butylated hydroxytoluene, was added before heating the samples. After cooling on ice, samples were centrifuged at $840 \mathrm{~g}$ for 15 minutes, and the absorbance of the supernatant was read at $532 \mathrm{~nm}$ (A532). Blanks for each sample were prepared and assessed in the same way to correct for the contribution of A532 to the sample. TBARS results were expressed as MDA equivalents by using 1,1,3,3-tetraethoxypropane as the standard.

2.5. Determination of mtDNA Copy Number. DNA samples were extracted from peripheral blood leukocytes. The mtDNA copy numbers were measured by real-time polymerase chain reaction (PCR) after correcting nuclear DNA levels. The forward and reverse primers complementary to nuclear $\beta$-actin gene were $5^{\prime}$-GAAATCGTGCGTGACATTAAAG- $3^{\prime}$ and $5^{\prime}$-ATCGGAACCGCTCATTG- $3^{\prime}$. The forward and reverse primers for mtDNA, which were complementary to the sequence of the mitochondrial ND1 gene, were $5^{\prime}$-ATTCTAGCCACATCAAGTCTTT- $3^{\prime}$ and $5^{\prime}$-GGAGGACGGATAAGAGGATAAT-3'. PCR was performed in a LightCycler 480 Real-Time PCR System (Roche Co., Mannheim, Germany), using LightCycler 480 SYBR Green I Master (Roche Co., Germany). DNA (10 ng) was mixed with $10 \mu \mathrm{L}$ SYBR Green PCR Master Mix containing $10 \mathrm{nmol}$ of forward and reverse primers, in a final volume of $20 \mu \mathrm{L}$. The PCR conditions were as follows: initial $50^{\circ} \mathrm{C} 2 \mathrm{~min}$, 
TABLE 1: Descriptive data of the children.

\begin{tabular}{|c|c|c|c|c|}
\hline Groups & $\begin{array}{l}\text { Normal } \\
(n=26)\end{array}$ & $\begin{array}{c}\mathrm{BA} \\
(n=26)\end{array}$ & $\begin{array}{c}\text { LT } \\
(n=26)\end{array}$ & $\begin{array}{c}\text { Hepatitis } \\
(n=6)\end{array}$ \\
\hline Age $(\mathrm{yr})($ mean \pm standard error $[\mathrm{SE}])$ & $2.1 \pm 1.5$ & $1.9 \pm 0.8$ & $8.6 \pm 0.9$ & $2.4 \pm 1.2$ \\
\hline Sex (male/female) & $1.1: 1$ & $1.1: 1$ & $0.8: 1$ & $0.8: 1$ \\
\hline \multicolumn{5}{|l|}{ Physical signs, number (\%) } \\
\hline Jaundice & $0(0)$ & $26(100)$ & $0(0)$ & $6(100)$ \\
\hline Edema of the lower limbs & $0(0)$ & $0(0)$ & $0(0)$ & $0(0)$ \\
\hline Hepatomegaly & $0(0)$ & $8(30.7)$ & $3(11.5)$ & $2(33.3)$ \\
\hline Splenomegaly & $0(0)$ & $11(42.3)$ & $3(11.5)$ & $1(16.7)$ \\
\hline Ascites & $0(0)$ & $2(7.6)$ & $0(0)$ & $0(0)$ \\
\hline \multicolumn{5}{|l|}{ Liver function tests (mean \pm SE) } \\
\hline $\operatorname{bil}(\mathrm{D})(\mathrm{mg} / \mathrm{dL})$ & $0.2 \pm 0.1^{* *}$ & $4.4 \pm 0.8^{*}$ & $0.3 \pm 0.1^{* *}$ & $3.4 \pm 0.9^{*}$ \\
\hline $\operatorname{bil}(\mathrm{T})(\mathrm{mg} / \mathrm{dL})$ & $0.50 \pm 0.2^{* *}$ & $6.5 \pm 1.2^{*}$ & $0.8 \pm 0.1^{* *}$ & $5.1 \pm 1.3^{*}$ \\
\hline AST (U/L) & $35.8 \pm 3.8^{* *}$ & $163.4 \pm 30.5^{*}$ & $32.7 \pm 5.7^{* *}$ & $341.9 \pm 177.3^{*}$ \\
\hline $\operatorname{ALT}(\mathrm{U} / \mathrm{L})$ & $21.4 \pm 5.6^{* *}$ & $121.8 \pm 26.4^{*}$ & $36.5 \pm 6.4^{* *}$ & $184.0 \pm 110.9^{*}$ \\
\hline ALP (U/L) & $150.3 \pm 15.4^{* *}$ & $510.0 \pm 81.3^{*}$ & $288.5 \pm 25.8^{* *}$ & $314.0 \pm 152.0^{*}$ \\
\hline Albumin (g [\%]) & $4.1 \pm 0.2$ & $3.0 \pm 0.6$ & $4.0 \pm 0.4$ & $3.1 \pm 0.8$ \\
\hline
\end{tabular}

BA: biliary atresia before liver transplantation; LT: biliary atresia after liver transplantation; bil(D): direct bilirubin; bil(T): total bilirubin; AST: aspartate aminotransferase; ALT: alanine aminotransferase; ALP: alkaline phosphatase. All results represent means \pm standard error $(\mathrm{SE}) .{ }^{*} P<0.05$ versus normal; ${ }^{* *} P<0.05$ versus BA.

$95^{\circ} \mathrm{C} 1 \mathrm{~min}, 40$ cycles of denaturation at $95^{\circ} \mathrm{C}$ for $15 \mathrm{~s}$, annealing at $60^{\circ} \mathrm{C}$ for $20 \mathrm{~s}$, and primer extension at $72^{\circ} \mathrm{C}$ for $15 \mathrm{~s}$, final $25^{\circ} \mathrm{C}$. The threshold cycle number $(\mathrm{Ct})$ values of the $\beta$-actin gene and the mitochondrial ND1 gene were determined for each individual quantitative PCR run. Ct values were used as a measure of the input copy number, and $\mathrm{Ct}$ value differences were used to quantify mtDNA copy number relative to the $\beta$ actin gene according to the following equation: relative copy number $(\mathrm{Rc})=2^{\Delta \mathrm{Ct}}$, where $\Delta \mathrm{Ct}$ is $\mathrm{Ct}_{\beta \text {-actin }}-\mathrm{Ct}_{\text {mtDNA }}$ [18]. Each measurement was performed at least 3 times and was normalized in each experiment against a serial dilution of a control DNA sample.

2.6. Statistical Analysis. SPSS for Windows version 13.0 (SPSS Inc., Chicago, IL, USA) was used for statistical analysis. Continuous variables were analyzed by independent $t$-test or ANOVA. The correlation of total bilirubin levels and oxidative stress and antioxidant enzyme activity was performed using Spearman's correlation analysis. Data are presented as means \pm SE. $P<0.05$ was considered statistically significant. The sample size was calculated with PASS sample size software, the two-sided confidence level $>0.95, P<0.05$, and power value $>0.8$ with $\alpha$ level $<0.05$.

\section{Results}

3.1. Patients' Clinical Data. This study included 26 normal healthy controls, 26 post-Kasai operation BA patients before and after LT, and 6 acute hepatitis patients. The descriptive data of the children included in this study are shown in Table 1. The liver function and levels of AST, ALT, and bilirubin were increased in the BA and acute hepatitis groups compared with the normal control group and significantly

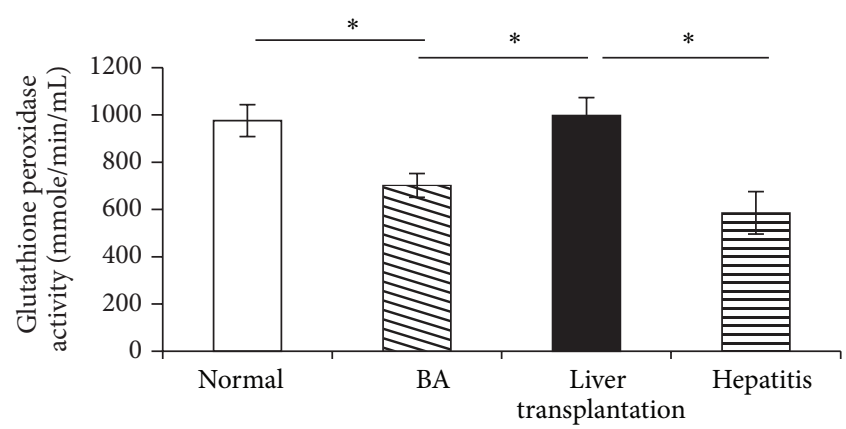

FIGURE 1: The activity of the antioxidant glutathione peroxidase in plasma was significantly decreased in the biliary atresia group (BA) before liver transplantation (LT) compared with the normal control group and significantly increased after undergoing liver transplantation in the LT group compared with the BA group, almost reaching the level of the normal control group. Data represent the mean \pm SE. ${ }^{*} P<0.05$ versus BA.

decreased in the LT group compared with the BA group (Table 1).

3.2. Oxidative Stress and Antioxidant Enzyme Activity. The activity of the antioxidant GPx significantly increased in the LT group compared with the BA group, almost reaching the level in the normal control group and significantly decreased in the BA group compared with the normal controls (Figure 1).

The expression of glutathione (GSH) is significantly increased in the LT group compared with the BA group, almost reaching the level in the normal control group and significantly decreased in the BA group compared with the normal control group (Figure 2(a)). The GSH/glutathione 


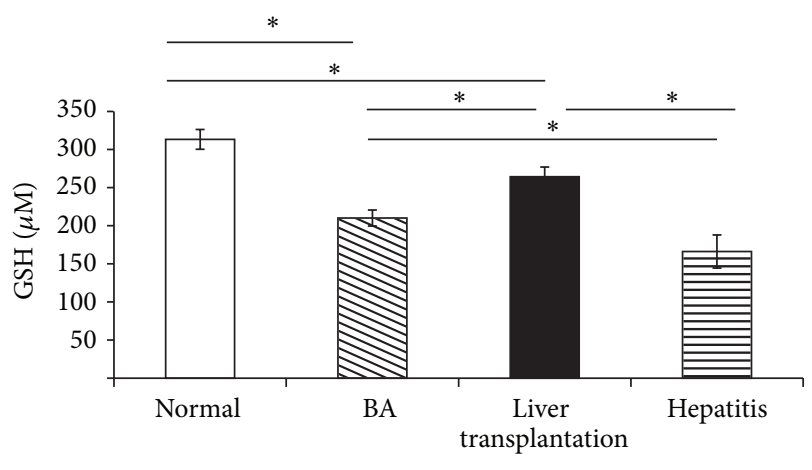

(a)

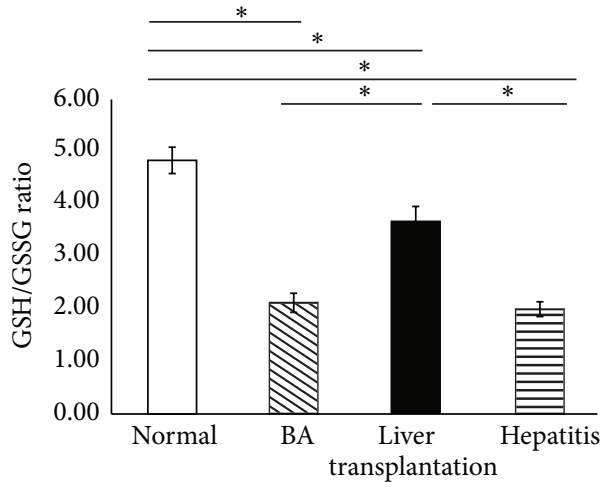

(b)

FIGURE 2: The expression of (a) total glutathione (GSH) and (b) the GSH/glutathione disulfide (GSSG) ratio in plasma significantly decreased in the biliary atresia group (BA) before liver transplantation (LT) compared with the normal control group and significantly increased after undergoing liver transplantation in the LT group compared with the BA group, almost to the level of the normal control group. Data represent the mean \pm SE. ${ }^{*} P<0.05$ versus BA.



FIGURE 3: The expression of catalase in plasma is significantly decreased in the biliary atresia (BA) group before liver transplantation (LT) compared with the normal control group and increased after undergoing LT. Data represent the mean \pm SE. ${ }^{*} P<0.05$ versus BA.

disulfide ratio significantly increased in the LT group compared with the BA group (Figure 2(b)).

The expression of CAT significantly increased in the LT group compared with the BA group and decreased in the BA group compared with the normal control group (Figure 3, Table 2).

The MDA level decreased in the LT group compared with the BA and hepatitis groups (Figure 4).

3.3. Correlations between the Severity of Cholestasis and Oxidative Stress and Antioxidant Enzyme Activity. The severity of cholestasis is directly related to the oxidative stress level and inversely related to the antioxidative level [19]. The total bilirubin level significantly correlated with the levels of MDA $(r=0.507, P<0.001), \mathrm{GPx}(r=-0.440, P=0.002)$,



FIGURE 4: The malondialdehyde (MDA) level in plasma decreased in patients after liver transplantation (LT) compared with biliary atresia (BA) patients before LT and the hepatitis group. Data represent the mean \pm SE. ${ }^{*} P<0.05$ versus BA.

TABLE 2: Comparison of liver supernatant (randomly selected cases) between mean levels $( \pm \mathrm{SE})$ of glutathione peroxidase and catalase (CAT) enzymes in different cholestasis groups and control children.

\begin{tabular}{lcc}
\hline Variable & GPx $(\mathrm{nmol} / \mathrm{min} / \mathrm{mL})$ & CAT $(\mathrm{nmol} / \mathrm{min} / \mathrm{mL})$ \\
\hline Normal $(n=3)$ & $67.01 \pm 6.32^{*}$ & $10.57 \pm 2.37^{*}$ \\
BA $(n=10)$ & $50.05 \pm 4.26$ & $7.11 \pm 1.53$ \\
LT $(n=10)$ & $72.48 \pm 5.21^{*}$ & $12.00 \pm 1.34^{*}$ \\
Hepatitis $(n=4)$ & $49.01 \pm 3.34$ & $10.26 \pm 1.62$ \\
\hline
\end{tabular}

BA: biliary atresia before liver transplantation; CAT: catalase; GPx: glutathione peroxidase; LT: biliary atresia after liver transplantation; ${ }^{*} P<0.05$ versus BA.

and GSH $(r=-0.478, P=0.001)$. The total bilirubin level significantly correlated with the levels of AST $(r=0.612$, $P<0.001)$ and ALT $(r=0.467, P=0.001)$. However, the correlation between total bilirubin and CAT levels $(r=$ $-0.022, P=0.887)$ and mtDNA copy number $(r=0.006$, $P=0.971)$ was not significant. 


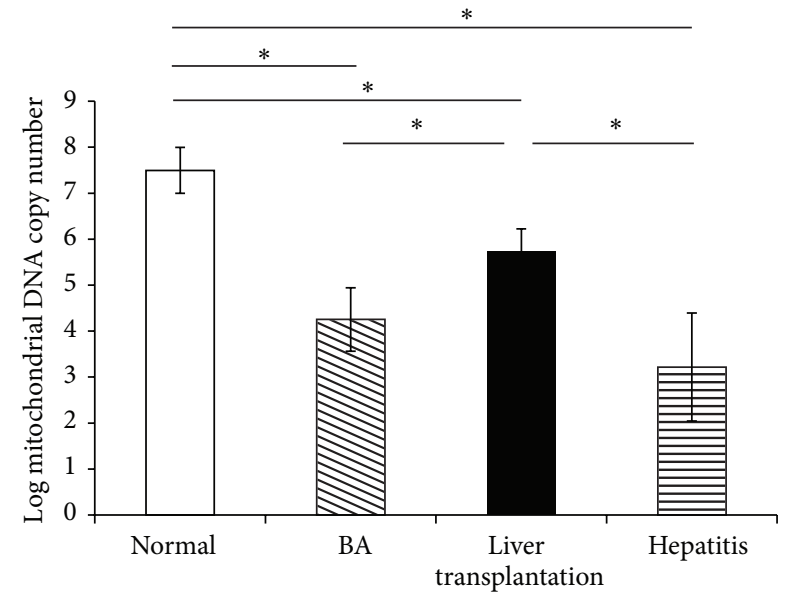

FIgURE 5: Comparison of the mitochondrial DNA (mtDNA) copy number in plasma between patients with biliary atresia (BA) and those receiving liver transplantation (LT), using the relative copy number $(\mathrm{Rc})=2^{\Delta \mathrm{Ct}}$, where $\Delta \mathrm{Ct}$ is $\mathrm{Ct}_{\beta \text {-actin }}-\mathrm{Ct}_{\mathrm{mtDNA}}$. The mtDNA copy number increased in the LT group compared with the BA group. Data represent the mean $\pm \mathrm{SE} .{ }^{*} P<0.05$ versus BA.

3.4. Post-LT mtDNA Copy Numbers. The mtDNA copy numbers were compared between the BA and LT groups by calculating the relative copy number $(\mathrm{Rc})=2^{\Delta \mathrm{Ct}}$, where $\Delta \mathrm{Ct}$ is $\mathrm{Ct}_{\beta \text {-actin }}-\mathrm{Ct}_{\mathrm{mtDNA}}$. The $\mathrm{mtDNA}$ copy numbers increased in the LT group compared with the BA group (Figure 5).

\section{Discussion}

This study more clearly indicates that both the mitochondrial number and the level of oxidative stress are involved in BA and that both the number of mitochondria and their antioxidant enzymes or their activity recover after LT. Therefore, the manipulation of mitochondria-associated antioxidative activity may be important in the management of BA even after LT. A thorough understanding of the mitochondrial influence on disease occurrence and progression is therefore crucial for the treatment of BA patients.

BA-induced obstructive jaundice in children is an important clinical issue $[1,20,21]$ that is associated with high morbidity and mortality rates [1]. Bile flow impairment leads to progressive liver injury, fibrosis, and end-stage liver cirrhosis. If not treated properly and effectively, LT is required for survival in most cases [1]. The pathogenesis of this disease is largely undefined, and there are no effective strategies to prevent its development. Studies exploring the underlying mechanisms, in particular those investigating protein profiles, have been limited by the inability to investigate the biliary tree at the onset of disease and to trace the progression of bile duct obstruction at defined phases. Many studies have reported increased antioxidant enzyme levels in BA and other liver diseases $[9,22,23]$, although some demonstrated a decrease in antioxidant enzyme levels $[7,8]$. In this study, we evaluated the levels of several antioxidant enzymes and oxidative stress markers at different stages, with emphasis on the BA and post-LT conditions, and gained a clearer understanding of $\mathrm{BA}$ pathogenesis that is relevant to its management strategy.

Oxidative stress is a major pathogenic event that occurs in several liver disorders, ranging from metabolic to proliferative conditions, and is a major cause of liver damage during LT [24]. Numerous studies have shown that oxidative stress is involved in the pathogenesis of cholestasis [25] and lipid peroxidation is responsible for the tissue injury that occurs in this condition [26]. End-stage liver cirrhosis accounts for approximately one-third of patients referred for LT [25]. Several enzymatic (i.e., CAT and GPx) and nonenzymatic (i.e., GSH and MDA levels) markers of chronic oxidative stress in the liver are well known [24]. Patients with jaundice have significantly higher mean levels of plasma lipid peroxides such as MDA and bilirubin than patients without jaundice [27]. Impairment of bile flow is likely to result in the accumulation of toxic hydrophobic bile salts within the hepatocytes, interfering with electron transport with consequent $\mathrm{H}_{2} \mathrm{O}_{2}$ and superoxide $\left(\mathrm{O}_{2}{ }^{-}\right)$formation [9, $22,28]$. Identification of these markers will enable the early detection of liver diseases and will allow for monitoring the degree of liver damage, response to pharmacological therapies, and development of new therapeutic approaches $[24,29]$. Many studies in humans and in rat models have been conducted to clarify the relationship between oxidative stress and antioxidants in the liver. In humans, an increase in the production of free oxygen radicals activates a complex defense system. This system includes GPx, CAT, GSH, glutathione reductase, and vitamins [26]. Our study showed a significant decrease in GSH, GPx, and CAT activities in patients with BA compared with their activities in the control group, which recovered after LT. In line with Salem et al.'s study [7], the levels of CAT in hepatitis or liver cirrhosis were low and presumably related to the effects of oxidative stress [8]. A marked decrease in the antioxidant status may lead to excess oxygen free radical formation, which promotes pathological processes in the liver.

In Granot et al's study [29], the levels of oxidants and antioxidants did not differ between cyclosporine A- (CsA-) treated patients after LT and healthy controls. In our study, markedly higher oxidative stress levels were related to more severe cholestasis. CsA binds to lipoproteins, primarily lowdensity lipoprotein phospholipids, which are expected to be particularly susceptible to lipid peroxidation [29]. In this study, we determine whether the high antioxidative enzyme levels were induced by LT or by CsA. More research is needed to clarify this issue. The administration of suitable antioxidant therapy just before the reestablishment of blood flow to the graft is important to prevent plasma redox imbalance and to prevent or ameliorate post-LT cholestatic injury of the liver graft [30].

Mitochondria are the major supplier of energy in mammalian cells [31] and provide energy necessary for the damage repair and cellular survival in response to the exposure of the environment during disease processes such as oxidative stress [31]. To meet this energy demand, signals are transmitted to the nucleus for inducing mitochondrial proliferation and mtDNA amplification for the production of a high number of functional mitochondria. In this study, the effect of 
mitochondria-associated oxidative stress on the development of BA that recovered after LT was observed by examining the plasma redox state and the mtDNA mass. On the basis of our previous studies, oxidative stress and mitochondrial intrinsic apoptosis pathways are involved in liver cell death in the bile duct ligation-induced cholestatic rat model [20, 32, 33]. Early-stage BA is associated with augmented oxidative DNA, mtDNA damage, and a decrease in mitochondrial copy number relative to that observed in late-stage BA. Our study was in accordance with Asakawa's which described that postoperative patients with BA were under increased oxidative stress [17]. Our report also proved that the oxidative stress increased more in BA before Kasai operation than after the operation and both are higher than the normal control [2]. Antioxidant therapy might be necessary to decrease oxidative stress in postoperative patients with BA [17]. Thus, altered mitochondrial function and increase in mitochondrial numbers after LT, in BA pathogenesis, will provide detailed information about the effects of oxidative stress on the development of BA, which improves after LT. These findings also suggest that mitochondrial and oxidative stress variations could serve as useful biomarkers for early BA treatment in the future.

In conclusion, the current study demonstrates that GPx, CAT, and GSH levels are low in patients with BA cholestasis. The significant increase in their levels after LT may point to their role as key enzymes in the protection of the liver from the hazards of free radical reactions and may reflect the appropriate activity of antioxidant barrier enzymes as a response to increased oxidative stress. Increased knowledge of redox regulation may have important clinical ramifications for understanding the pathogenesis of BA liver diseases and for developing therapeutic approaches.

\section{Abbreviations}

$\begin{array}{ll}\text { BA: } & \text { Biliary atresia } \\ \text { CAT: } & \text { Catalase } \\ \text { GPx: } & \text { Glutathione peroxidase } \\ \mathrm{H}_{2} \mathrm{O}_{2}: & \text { Hydrogen peroxide } \\ \text { LT: } & \text { Liver transplantation } \\ \text { MDA: } & \text { Malondialdehyde } \\ \text { mDNAN: } & \text { Mitochondrial DNA } \\ \text { ROS: } & \text { Reactive oxygen species. }\end{array}$

\section{Conflict of Interests}

The authors declare no conflict of interests.

\section{Authors' Contribution}

Mao-Meng Tiao, Li-Tung Huang, and Chao-Long Chen conceived and designed the experiments. Ying-Hsien Huang and Kuo-Shu Tang performed the experiments. Chih-Jen Chen and Jiin-Haur Chuang analyzed the data. Chih-Jen Chen contributed reagents/materials/analysis tools. MaoMeng Tiao, Kuo-Shu Tang, and Chih-Jen Chen wrote the paper.

\section{Acknowledgment}

This work was supported by grants (CMRPG8A0111-3, CMRPG8D0901, CMRPG8B0131-2, and CMRPG8D0171) from the Chang Gung Memorial Hospital, Taiwan.

\section{References}

[1] C. H. Hsiao, M. Chang, H. Chen et al., "Universal screening for biliary atresia using an infant stool color card in Taiwan," Hepatology, vol. 47, no. 4, pp. 1233-1240, 2008.

[2] M. M. Tiao, T. K. Lin, F. Y. Kuo et al., "Early stage of biliary atresia is associated with significant changes in 8-hydroxydeoxyguanosine and mitochondrial copy number," Journal of Pediatric Gastroenterology and Nutrition, vol. 45, no. 3, pp. 329334, 2007.

[3] S. C. Xu, Y. B. Chen, H. Lin et al., "Damage to mtDNA in liver injury of patients with extrahepatic cholestasis: the protective effects of mitochondrial transcription factor A," Free Radical Biology and Medicine, vol. 52, no. 9, pp. 1543-1551, 2012.

[4] A. Favier, "Oxidative stress in human diseases," Annales Pharmaceutiques Francaises, vol. 64, no. 6, pp. 390-396, 2006.

[5] I. Moreno, S. Pichardo, A. Jos et al., "Antioxidant enzyme activity and lipid peroxidation in liver and kidney of rats exposed to microcystin-LR administered intraperitoneally," Toxicon, vol. 45, no. 4, pp. 395-402, 2005.

[6] J. Czuczejko, B. A. Zachara, E. Staubach-Topczewska, W. Halota, and J. Kedziora, "Selenium, glutathione and glutathione peroxidases in blood of patients with chronic liver diseases," Acta Biochimica Polonica, vol. 50, no. 4, pp. 1147-1154, 2003.

[7] T. A. Salem, M. F. El-Refaei, and G. A. Badra, "Study of antioxidant enzymes level and phagocytic activity in chronic liver disease patients," The Egyptian Journal of Immunology, vol. 10, no. 1, pp. 37-45, 2003.

[8] H. Togashi, H. Shinzawa, H. Wakabayashi et al., "Activities of free oxygen radical scavenger enzymes in human liver," Journal of Hepatology, vol. 11, no. 2, pp. 200-205, 1990.

[9] E. Broide, E. Klinowski, G. Koukoulis et al., "Superoxide dismutase activity in children with chronic liver diseases," Journal of Hepatology, vol. 32, no. 2, pp. 188-192, 2000.

[10] M. H. Hussein, T. Hashimoto, T. Suzuki et al., "Children undergoing liver transplantation for treatment of inherited metabolic diseases are prone to higher oxidative stress, complement activity and transforming growth factor-betal," Annals of Transplantation, vol. 18, no. 1, pp. 63-68, 2013.

[11] B. Halliwell, "Free radicals and antioxidants: a personal view," Nutrition Reviews, vol. 52, no. 8, pp. 253-265, 1994.

[12] K. Venardos, G. Harrison, J. Headrick, and A. Perkins, "Effects of dietary selenium on glutathione peroxidase and thioredoxin reductase activity and recovery from cardiac ischemiareperfusion," Journal of Trace Elements in Medicine and Biology, vol. 18, no. 1, pp. 81-88, 2004.

[13] M. Renis, P. Cantatore, P. L. Polosa, F. Fracasso, and M. N. Gadaleta, "Content of mitochondrial DNA and of three mitochondrial RNAs in developing and adult rat cerebellum," Journal of Neurochemistry, vol. 52, no. 3, pp. 750-754, 1989.

[14] J. Y. Jeng, T. S. Yeh, J. W. Lee, S. H. Lin, T. H. Fong, and R. H. Hsieh, "Maintenance of mitochondrial DNA copy number and expression are essential for preservation of mitochondrial function and cell growth," Journal of Cellular Biochemistry, vol. 103, no. 2, pp. 347-357, 2008. 
[15] C. Richter, V. Gogvadze, R. Laffranchi et al., "Oxidants in mitochondria: from physiology to diseases," Biochimica et Biophysica Acta-Molecular Basis of Disease, vol. 1271, no. 1, pp. 67-74, 1995.

[16] M. Najimi, X. Stéphenne, C. Sempoux, and E. Sokal, "Regulation of hepatic EAAT-2 glutamate transporter expression in human liver cholestasis," World Journal of Gastroenterology, vol. 20, no. 6, pp. 1554-1564, 2014.

[17] T. Asakawa, Y. Tanaka, K. Asagiri, H. Kobayashi, K. Tanikawa, and M. Yagi, "Oxidative stress profile in the post-operative patients with biliary atresia," Pediatric Surgery International, vol. 25, no. 1, pp. 93-97, 2009.

[18] M. M. Kim, J. D. Clinger, B. G. Masayesva et al., "Mitochondrial DNA quantity increases with histopathologic grade in premalignant and malignant head and neck lesions," Clinical Cancer Research, vol. 10, no. 24, pp. 8512-8515, 2004.

[19] A. S. Loginov, B. N. Matyushin, S. M. Chebanov, and V. L. Reshetnyak, "Cholestasis assessment by activity of antioxidant enzymes and composition of plasma lipoproteins in patients with hepatic diseases," Terapevticheskii Arkhiv, vol. 70, no. 4, pp. 40-42, 1998.

[20] Y. Akazawa and G. J. Gores, "Death receptor-mediated liver injury," Seminars in Liver Disease, vol. 27, no. 4, pp. 327-338, 2007.

[21] C. W. Lee, J. H. Chuang, P. W. Wang et al., "Effect of glucocorticoid pretreatment on oxidative liver injury and survival in jaundiced rats with endotoxin cholangitis," World Journal of Surgery, vol. 30, no. 12, pp. 2217-2226, 2006.

[22] M. Ono, C. Sekiya, M. Ohhira et al., "Elevated level of serum Mn-superoxide dismutase in patients with primary biliary cirrhosis: possible involvement of free radicals in the pathogenesis in primary biliary cirrhosis," Journal of Laboratory and Clinical Medicine, vol. 118, no. 5, pp. 476-483, 1991.

[23] N. A. Ismail, S. H. Okasha, A. Dhawan, A. O. Abdel-Rahman, O. G. Shaker, and N. A. Sadik, "Antioxidant enzyme activities in hepatic tissue from children with chronic cholestatic liver disease," Saudi Journal of Gastroenterology, vol. 16, no. 2, pp. 9094, 2010.

[24] L. Cesaratto, C. Vascotto, S. Calligaris, and G. Tell, "The importance of redox state in liver damage," Annals of Hepatology, vol. 3, no. 3, pp. 86-92, 2004.

[25] G. Alpini, J. M. McGill, and N. F. LaRusso, “The pathobiology of biliary epithelia," Hepatology, vol. 35, no. 5, pp. 1256-1268, 2002.

[26] L. Y. Tsai, K. T. Lee, S. M. Tsai, S. C. Lee, and H. S. Yu, “Changes of lipid peroxide levels in blood and liver tissue of patients with obstructive jaundice," Clinica Chimica Acta, vol. 215, no. 1, pp. 41-50, 1993.

[27] L. Y. Tsai, S. M. Tsai, K. T. Lee, and H. S. Yu, "Levels of plasma lipid peroxides before and after choledocholithotomy in patients with obstructive jaundice," Journal of UOEH, vol. 14, no. 4, pp. 261-269, 1992.

[28] S. Krähenbühl, S. Fischer, C. Talos, and J. Reichen, "Ursodeoxycholate protects oxidative mitochondrial metabolism from bile acid toxicity: dose-response study in isolated rat liver mitochondria," Hepatology, vol. 20, no. 6, pp. 1595-1601, 1994.

[29] E. Granot, H. Elinav, and R. Kohen, "Markers of oxidative stress in cyclosporine-treated and tacrolimus-treated children after liver transplantation," Liver Transplantation, vol. 8, no. 5, pp. 469-475, 2002.
[30] E. Serrano, J. Díaz, F. Acosta, P. Parrilla, and L. F. Carbonell "Plasma redox imbalance during reperfusion and early posttransplantation cholestasis parameters in human liver transplantation," Transplantation Proceedings, vol. 31, no. 6, pp. 23782379, 1999.

[31] H. C. Lee and Y. H. Wei, "Mitochondrial biogenesis and mitochondrial DNA maintenance of mammalian cells under oxidative stress," International Journal of Biochemistry and Cell Biology, vol. 37, no. 4, pp. 822-834, 2005.

[32] C. H. Ahn, E. G. Jeong, J. W. Lee et al., "Expression of beclin-1, an autophagy-related protein, in gastric and colorectal cancers," APMIS, vol. 115, no. 12, pp. 1344-1349, 2007.

[33] M. M. Tiao, T. K. Lin, C. W. Liou et al., "Early transcriptional deregulation of hepatic mitochondrial biogenesis and its consequent effects on murine cholestatic liver injury," Apoptosis, vol. 14, no. 7, pp. 890-899, 2009. 


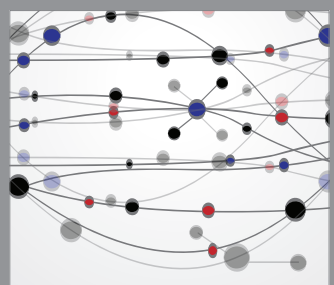

The Scientific World Journal


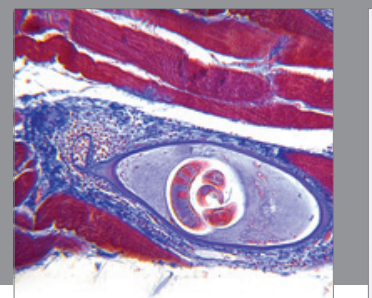

Gastroenterology

Research and Practice
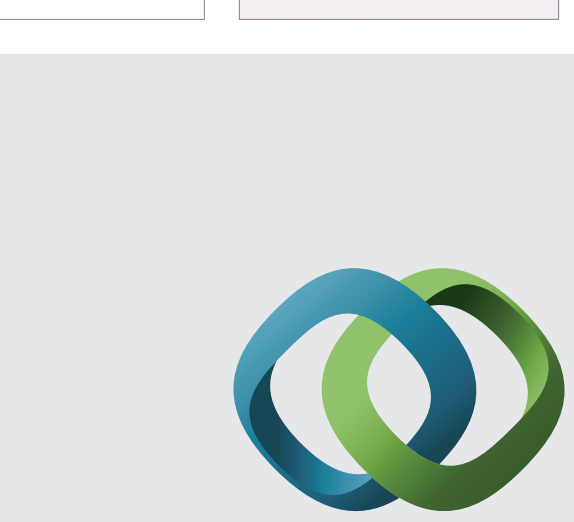

\section{Hindawi}

Submit your manuscripts at

http://www.hindawi.com



Disease Markers
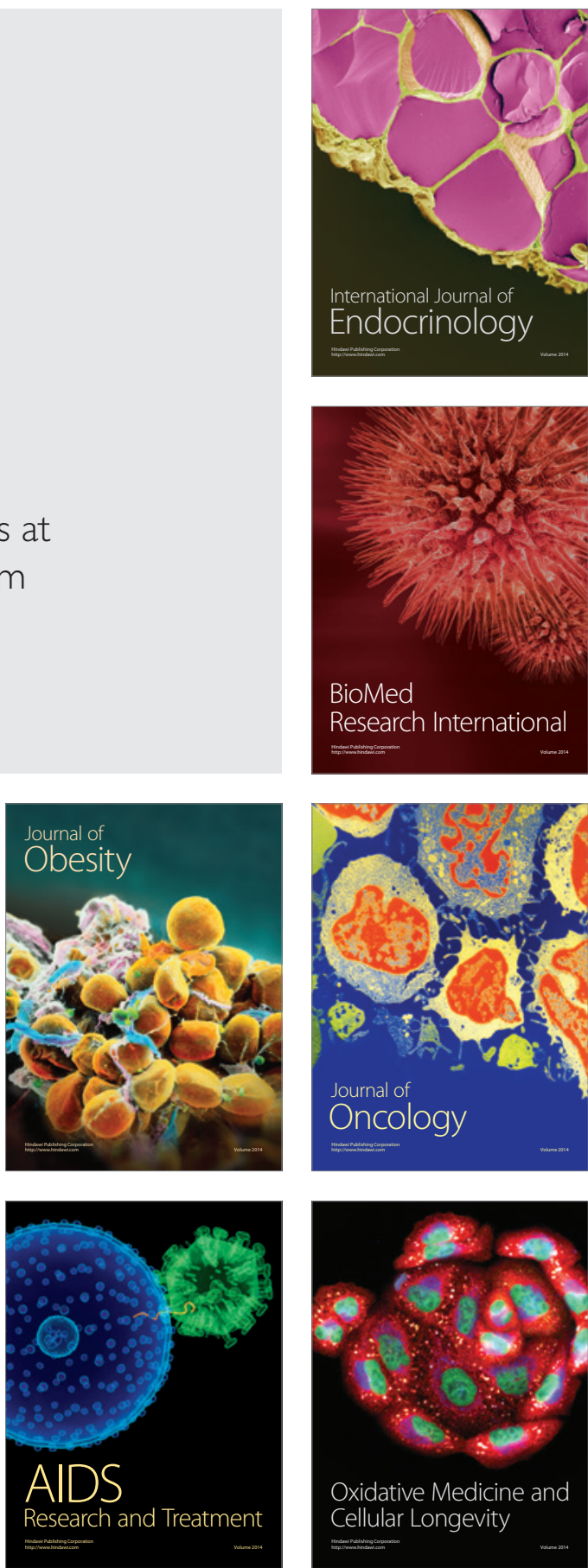